\title{
Impedance therapy in rehabilitation of degenerative disc disease
}

\author{
Kostka $\mathrm{P}^{1}$, Ziakova $\mathrm{E}^{2}$ \\ Institute of Impedance Therapy, Bratislava, Slovakia. pavol.kostka.dr@gmail.com
}

\begin{abstract}
AIM: The aim of this work was to investigate the effects of Impedance Therapy (IT) in the treatment of degenerative disc disease (DDD) by confirming the presence of the "disc grow-up" (DGU) phenomenon. METHOD: The set consisted of 55 patients with DDD with the average age of 51.3 years divided into two groups - the experimental group and the control group. The experimental group consisted of 29 patients with the average age of 56.7 years. The control group consisted of 26 patients with the average age of 45.8 years.

RESULTS: In the experimental group of patients with DDD, who received IT, the DGU phenomenon with a success rate of $76 \%$ was observed, with the average increase in the volume of the intervertebral disc of $31 \%(p<0.000)$. In the control group of patients receiving the standard electrotherapy, the DGU phenomenon was not proven - the DDD progressed normally with the mean volume reduction of $15 \%$ $(p<0.000)$.

CONCLUSIONS: Degenerative disc disease, as a disease of modern civilization, is treatable. It can be concluded that the theory that degenerative disc changes are irreversible was disproven by the impact of impedance therapy (Tab. 5, Ref. 14). Text in PDF www.elis.sk.

KEY WORDS: impedance therapy, specific electrical impulse, DGU phenomenon, "Disc grow-up",
\end{abstract} degenerative disc disease.

\section{Introduction}

The category of civilization diseases involving spine changes due to degenerative disc disease are now considered to be the second most common cause of being not capable to work in industrialized countries and is the most common cause of disability for people of productive age. Epidemiological studies showed that up to $80 \%$ of people need a medical assistance to treat vertebrogenic pain at least once during their lifetime (12). In $5 \%$ to 10 $\%$, an acute pain syndrome evolved into a chronic condition (3). Chronic back pain has serious social and economic consequences. Approximately $75 \%$ of the total financial costs spent on back pain treatment (8) are used to treat chronic pain.

\section{Theoretical background}

In the clinical investigation of the method of joining and healing small vessels in the past, we focused also on the histological analysis of the site of vascular junction. We found that under the cauterization currents, the healing of the observed vessels pro-

${ }^{1}$ Institute of Impedance Therapy, Bratislava, Slovakia, and ${ }^{2}$ Department of Physiotherapy, Institute of Physiotherapy, Balneology and Medical Rehabilitation, University of Ss. Cyril and Methodius in Trnava, Slovakia

Address for correspondence: P. Kostka, MD, Klinika impedančnej terapie, Černyševského 26, SK-851 01 Bratislava, Slovakia.

Phone: +0905743554 ceeded differently in comparison to heeling without these currents. Consequently, we focused on the effect of current and voltage on the regenerating part of the tissue. The results of the study were more than satisfactory and therefore we began to focus on the more general options of using this mechanism to regenerate the human body. In the following investigation, we examined the effects of electrical impulses on back pain caused by degenerative changes in the spine. For this purpose, we built a pulse generator. Then, we tested the therapeutic effect of generated impulses to painful conditions of the lumbar spine caused by degenerative disc disease. We currently own the 7th generation generator. The result of the testing was that the painful spine condition was alleviated by the impulse, which changes the properties of the skin by making it more conductive. The neurophysiological basis of the impulseinduced change in skin resistance is called the psychogalvanic reflex (PGR). Galvanic reaction of the skin leads to an increase in the electrical conductivity (resistance reduction) of the skin. The mechanism of the PGR reflex is associated with the activation of sweat glands via postganglionic sympathetic fibers. Thus, sweat becomes an electrolytic conductor and indirectly changes the electrical properties of the skin cover $(2,13)$.

\section{Specific Electrical Impulse - SEI}

Based on sweat conductivity measurements, we created a specific electrical impulse, SEI, to stimulate people with vertebrogenic pain caused by degenerative disc disease. In the set of patients, we analyzed the organism's responses to the applied SEI. 
137-142

Conditions of SEI application:

- there are time pauses and amplitude changes during the impulse application,

- the chemical composition of the sweat changes during the application,

- combination with dry needle therapy (effect on skin resistance change).

Based on the above-mentioned conditions for SEI application, we defined the impulse sequence, which, while maintaining PGR reflex induction, can have a beneficial effect on pain in degenerative disc disease. The analysis and subsequent synthesis of stimulation results enabled the creation of a new treatment method, so-called impedance therapy, which leads to the most effective removal of vertebrogenic pain in a patient with DDD.

\section{“Disc grow-up" (DGU)}

In 2009 (after 10 years of SEI application) we started to investigate, monitor and evaluate structural changes of the spine based on CT and MRI examinations. After evaluating the findings in more than 1000 patients, radiologists confirmed that structural changes occured in the spine of treated patients. These changes mainly concerned the size of the intervertebral disc, namely the volume and size of the herniated structures of the disc. By converting DICOM images into 3D visualization, it was confirmed that the change in the volume of intervertebral discs depends on the SEI composition in combination with dry needle therapy. Following this method, we introduced the term "disc grow-up" (DGU) phenomenon as a manifestation of regeneration and healing of the intervertebral disc. The DGU phenomenon was confirmed in 1078 patients based on 3D visualization (6).

\section{Material and methods}

The set consisted of 55 patients with an established diagnosis according to the International Classification of Diseases G54.0, 1, 2, 4 and M54, 2, 4, 5, 12, 16, 17 with the average age of 51.3 years. The set was divided into two groups - the experimental group and the control group. Totally, 61 patients were enrolled in the clinical trial, 6 of whom were excluded ( 4 on the basis of exclusion criteria and 2 decided to terminate the study). The first group, i.e., the experimental group, consisted of 29 patients with the average age of 56.7 years, of which 22 were men with the average age of 57.2 and 7 were women with the average age of 55.2. The second group, i.e., the control group, consisted of 26 patients with the average age of 45.8 years, of which 10 were women with the average age of 45.8 years and 16 were men with the average age of 45.9 years.

\section{Inclusion criteria}

Age 18 to 80, regardless of sex.

The patient understands and voluntarily signs the informed consent with a medical observation prior to any medical observation, or procedure and after explaining its nature and the purpose of observation.
The patient accepts the conditions, scope and the nature of the medical observation with their signature.

The patient has been treated for pain syndrome for 6 months or longer and differentially diagnosed with a degenerative disc disease, where degenerative disc disease was proven, without further questioning, to be the evident cause of the pain.

The patient is willing and able to cooperate and has agreed to all conditions of their participation in medical observation.

\section{Exclusion criteria}

Age under 18 years and over 80 years at the time of inclusion in the medical observation.

The presence of recurrent upper respiratory tract diseases (hereinafter referred to as HDC), i.e., repeated treatment of upper respiratory tract infections with antibiotics with a minimum interval of 6 months prior to inclusion in the medical observation. - Has undergone tissue or organ transplantation.

- An unspecified fever condition with no known cause at least 3 months prior to the start of medical observation.

- Deterioration of health with a proven sequestrum.

- Diagnosed discitis.

- Newly discovered paroxysmal supraventricular tachycardia with extrasystoles.

- Poorly compensated arrhythmias and arrhythmia attacks lasting longer than 2 days.

- Long bone fracture.

- Newly discovered, medically treated blood clotting disorder.

- Hypertensive crisis.

- Sudden diabetes mellitus.

- Sudden psychiatric illness or recurrence of psychosis.

- Epileptic seizure in last 3 months.

- Implanted osteosynthetic material that has been rejected.

- Implanted pacemaker.

- History of anaphylactic reactions or severe reactions to certain blood derivatives.

- Previously diagnosed with hepatitis B or C.

- Pregnant or nursing mothers.

- History of chronic alcoholism or illicit drug abuse at least 12 months prior to inclusion in this study.

- Cancer.

\section{Reasons for interrupting the participation in the clinical trial}

The patient withdraws an informed consent to participate in medical observation.

It has been additionally discovered that the patient does not meet all inclusion criteria for inclusion in the medical observation.

It has been additionally discovered that the patient meets one of the exclusion criteria for exclusion from the medical observation.

The patient is unable to adhere to the main medical observation conditions.

1. they do not adhere to the specified therapy interval

2. they do not adhere to the individual treatment regimen for an acute recurrence of pain

3. they do not adhere to the individual treatment regimen for an acute febrile illness 
4. they do not adhere to the individual treatment regimen for other noninfectious diseases/difficulties (injury, car crash, mental illness)

5. they do not adhere to the individual treatment regimen for newly diagnosed skin disease

6. they do not adhere to an individual treatment regimen for infectious disease

The investigator additionally finds any reason in the context of simulation or dissimulation of the painful condition.

Any information, fact, belief, and so on prevent the individual from taking part in the medical observation.

A randomized clinical trial took place from October 2016 to May 2018. The aim of the study was to investigate the impact of innovative IT on improving the health condition during the rehabilitation of DDD patients; to verify the impedance therapy for the treatment of degenerative disc disease as a civilization disease. The aim of the study was to monitor the effect of IT as a drug-free therapy of degenerative disc disease through objective MRI spine findings and neurological examinations, and subjective patient experience before and after completion of a given treatment course. Patients were included in the long-term rehabilitation plan anonymously for approximately 5 to 10 months. The rehabilitation plan had a common pattern for both groups, while one (experimental) group received electrotherapy with SEI and the second (control) group received standard electrotherapy. Patients were registered in an information system anonymously and were not informed which group they belonged to. To this end, they were assigned personalized RFID chips (providing an anonymous process and protected access to patient data in the patient information system).

\section{Methods for assessing the effectiveness of the applied electro-} therapy

Numeric Rating Scale (NRS) (5)

MRI examination - to assess and compare the effect of therapy, MR images in DICOM format were processed to realize 3D visualization. Then, the DGU phenomenon was evaluated as a manifestation of the regeneration, recovery and growth of the intervertebral disc. The acquired MR images were processed in In Vesalius (11). The examination was evaluated by a neurologist, radiologist and neurosurgeon. Standardized volume measurements were performed on each of the magnetic resonance devices prior to the start of the study. The reference volume created by us was recorded on a magnetic resonance device and a $3 \mathrm{D}$ reconstruction was created. Thus, the proof of the accuracy of the magnetic resonance device was obtained. The standard deviation of the magnetic resonance device was about $\pm 10 \%$. For our purposes, a tolerance of $\pm 0.68 \%$ at the precision level was accepted.

Neurological examination of periosteal and tendon reflexes (PTR) 7-point scale, where: 0 - reflexes not manifested, 3 -average reflexes, 6 - strong reflexes.

Blood lactate level - supplemental measurement. One of the factors of fatigue is the level of lactate in the blood, the increased concentration of which is the cause of the metabolic acidification of the internal environment of the body, which is also manifested by a decrease in performance. During our study, lactate levels in the blood were measured at rest and during exercise. The measurement results are part of the CRF of the study and control group of patients. The mean blood lactate levels were for exercise of 10 to $30 \mathrm{~min}$. - column $\mathrm{N}$ (before and after inclusion in the study). Blood lactate levels at rest were from 0.7 to $1.8 \mathrm{mmol} / \mathrm{L}$ and 4 $\mathrm{mmol} / \mathrm{L}$ level of lactate in the blood during exercise is generally considered to be the limit of the body's effectiveness during exercise. Measuring the level of blood lactate provides us with information on the impact of this innovative method on improving body condition. In the experimental group, the optimization of blood lactate levels was on average $30 \%$ better than in the control group. In the experimental group, blood lactate returned to physiological values in all the patients in the study group, in which the DGU phenomenon was proven.

The outcome of the treatment was considered successful when simultaneously:

- conversion of DICOM images from MRI examinations before and after completion of the rehabilitation plan confirmed morphological changes of the intervertebral discs - the DGU phenomenon

- comparative neurological examination showed an improvement

- the patient considered their subjective condition to have improved after losing or significantly reducing algic complications, allowing them to return to their original activities, not only self-care activities.

\section{Rehabilitation}

The long-term rehabilitation plan was based on a retrospective analysis of 9,831 patients, who underwent totaly 248,643 impedance therapies (7).

It consists of blocks and their associated phases:

1st block - aimed at reducing pain - according to a rehabilitation plan - applying standard physiotherapy procedures in combination with electrotherapy

Phase 1 - classification, Phase 2 - RT symptom (retrospective symptom), Phase 3 - inclusion of individual exercise

2nd block - aimed at increasing physical performance Phase 4 -inclusion of training, Phase 5-metabolism analysis, Phase 6 - regeneration fixation

3rd block - non-medical process (repeated exercise measurements with defining blood lactate levels in the context of heart rate, maintaining proper exercise, maintaining the right weight)

The observed patients underwent rehabilitation three times in two weeks in a total number of therapies ranging from 29 to 48 .

\section{Tab. 1. Rehabilitation plan - control group.}

\begin{tabular}{lccc}
\hline 1. & Low-frequency currents therapy -5 times \\
\hline $1 \mathrm{x}$ & diadynamic & $\mathrm{DF}$ & $8-10 \mathrm{mins}$ \\
$2 \mathrm{x}$ & diadynamic & $\mathrm{LP}$ & $17-19 \mathrm{mins}$ \\
$3 \mathrm{x}$ & TENS & $2-8 \mathrm{~Hz}$ & $12-15 \mathrm{mins}$ \\
\hline 2. & \multicolumn{2}{c}{ Medium frequency and interference currents therapy -5 times } \\
\hline
\end{tabular}


The experimental group of patients underwent rehabilitation with IT and the control group underwent the same rehabilitation plan with the exception that a classical electrotherapy impulse in form of 10 therapies was applied instead of SEI:

Each implemented therapy was recorded in the information system; it lasted from 90 to $120 \mathrm{~min}$. and consisted of:

1. examination of the patient, recording their medical condition

2. application of electrotherapy - SEI or classic electrotherapy

3. application of thermal and light therapy, application of dry needle therapy

4. manual therapy

5. patient examination after electrotherapy

All the data obtained from the enrolment to the termination of participation in the study were recorded in a summarized anonymized case report (CRF) for the patient. The anonymized summary of the results of patients in the experimental and the control group before and after inclusion in the rehabilitation plan at the level of the first block of impedance therapy forms an annex to this document.

\section{Methods of safety monitoring}

Local and systemic tolerance of patients to medical procedures was observed individually using the adverse event monitoring protocols. The patient was under the direct control of medical personnel during therapy. They were exposed to physical stress during electrotherapy. Physical stress is the basis of the therapeu- tic effects of electrotherapy and is approved by the state regulatory authority.

\section{Statistical analysis}

The comparison of the results in individual patients was based on anonymized patient data, to which the final CRF composition was subordinated. We verified the intervertebral disc volume evaluation by the accuracy of the magnetic resonance device based on sample calibration volume measured in each magnetic resonance device.

The data obtained were processed using descriptive statistics and the Kolmogorov-Smirnov and Shapiro-Wilk tests were used to evaluate normal data distribution. If the significance of the alpha level test was less than 0.05 , this was a selection with a disrupted normal data distribution and therefore, nonparametric statistics tools were used for evaluation - the Wilcoxon pair test. To obtain the standard size measure, the Effect size was calculated, which, together with the significance value, gave us information about the magnitude and significance of the effect.

\section{Ethical approval of the biomedical study}

Ethical approval took place in 2012, when the methodological process for the implementation of a clinical study was prepared in cooperation with the Faculty of Pharmacy of Comenius University in Bratislava under the supervision of Professor PharmDr. J. Kyselovič, CSc. The implementation of the study was in line with the guidelines set out in the Helsinki Declaration (14). All patients agreed to be enrolled in the study and with the anonymized data

Tab. 2. Statistical analysis of the experimental group.

\begin{tabular}{|c|c|c|c|c|c|c|c|c|}
\hline Experimental group & $\mathrm{n}$ & Mean & SD & Min & $\operatorname{Max}$ & $\mathrm{Z}$ & $\mathrm{p}$ & $\mathrm{r}$ \\
\hline $\mathrm{cm} 3$ before & 29 & 8.0394 & 6.23834 & 0.84 & 23.14 & -4.703 & 0.000 & -0.873 \\
\hline $\mathrm{cm} 3$ after & 29 & 10.530 & 7.74621 & 1.11 & 26.78 & & & \\
\hline pain input & 29 & 6.41 & 0.983 & 5 & 8 & -4.739 & 0.000 & -0.880 \\
\hline pain_output & 29 & 1.45 & 0.47 & 1 & 2 & & & \\
\hline PTR_input & 29 & 1.86 & 1.187 & 1 & 5 & -2.886 & 0.004 & -0.536 \\
\hline PTR_output & 29 & 2.59 & 0.501 & 2 & 3 & & & \\
\hline
\end{tabular}

$\mathrm{Z}$ - Wilcoxon test calculation; $\mathrm{p}$ - value of statistical significance; $\mathrm{r}$ - effect size

Tab. 3. Statistical analysis of the control group.

\begin{tabular}{|c|c|c|c|c|c|c|c|c|}
\hline Control group & $\mathrm{n}$ & Mean & SD & Min & Max & Z & $\mathrm{P}$ & $\mathrm{r}$ \\
\hline $\mathrm{cm} 3$ before & 26 & 10.9145 & 7.70878 & 1.61 & 27.12 & -4.45735 & 0.000 & -0.874 \\
\hline $\mathrm{cm} 3$ after & 26 & 9.2905 & 6.51451 & 1.45 & 23.24 & & & \\
\hline pain_input & 26 & 5.69 & 0.884 & 4 & 7 & -4.542 & 0.000 & -0.891 \\
\hline pain_output & 26 & 3.77 & 0.652 & 2 & 5 & & & \\
\hline PTR_input & 26 & 2.77 & 1.608 & 0 & 6 & 0.000 & 1.000 & 0.000 \\
\hline PTR_output & 26 & 2.77 & 1.478 & 0 & 5 & & & \\
\hline
\end{tabular}

$\mathrm{Z}$ - Wilcoxon test calculation; $\mathrm{p}$ - value of statistical significance; $r$ - effect size

Tab. 4. Statistical calculation for lactate measurements in the experimental group.

\begin{tabular}{|c|c|c|c|c|c|c|c|c|}
\hline \multirow{3}{*}{ Experimental group - lactate } & \multicolumn{5}{|c|}{ Paired Differences } & \multirow{3}{*}{$\mathrm{t}$} & \multirow{3}{*}{ df } & \multirow{3}{*}{$\mathrm{p}$} \\
\hline & \multirow{2}{*}{ Mean } & \multirow{2}{*}{ SD } & \multirow{2}{*}{$\begin{array}{c}\text { Std. Error } \\
\text { Mean }\end{array}$} & \multicolumn{2}{|c|}{$95 \%$ Confidence Interval of the Difference } & & & \\
\hline & & & & Lower & Upper & & & \\
\hline Pair 1 aero_in - aero_out & 2.4172 & 1.3472 & 0.2502 & 1.9048 & 2.9297 & 9.662 & 28 & 0.000 \\
\hline Pair 2 rest_in - rest_out & 1.6690 & 0.5813 & 0.1079 & 1.4478 & 1.8901 & 15.461 & 28 & 0.000 \\
\hline
\end{tabular}

$\mathrm{t}$ - t-test calculation, $\mathrm{p}$ - value of statistical significance, $\mathrm{df}$ - degree of freedom 
Tab. 5. Statistical calculation of lactate in control group.

\begin{tabular}{|c|c|c|c|c|c|c|c|c|}
\hline \multirow{3}{*}{ Control group - lactate } & \multicolumn{5}{|c|}{ Paired Differences } & \multirow{3}{*}{$\mathrm{t}$} & \multirow{3}{*}{ df } & \multirow{3}{*}{$\mathrm{p}$} \\
\hline & \multirow{2}{*}{ Mean } & \multirow{2}{*}{$\mathrm{SD}$} & \multirow{2}{*}{$\begin{array}{l}\text { Std. Error } \\
\text { Mean }\end{array}$} & \multicolumn{2}{|c|}{$95 \%$ Confidence Interval of the Difference } & & & \\
\hline & & & & Lower & Upper & & & \\
\hline Pair 1 aero in - aero out & 0.9885 & 1.1782 & 0.2311 & 0.5126 & 1.4644 & 4.278 & 25 & 0.000 \\
\hline Pair 2 rest_in - rest_out & -0.0231 & 0.2747 & 0.0539 & -0.1340 & 0.0879 & -0.428 & 25 & 0.672 \\
\hline
\end{tabular}

$\mathrm{t}$ - $\mathrm{t}$-test calculation, $\mathrm{p}$ - value of statistical significance, $\mathrm{df}$ - degree of freedom

processing. They were allowed to terminate the participation in the clinical trial at any time.

\section{Results}

The anonymized final summary of CRF of patients comparing MRI results before and after being included in the rehabilitation plan at the level of the first block of impedance therapy of the first and second group of patients form annexes to this document. IT in the patient group caused changes in health that copied the course of changes in the rehabilitation plan. Patients enrolled in the experimental group had a period of RT symptoms (retrospective symptoms) - occurrence of past difficulties with a lower intensity. The presence of RT symptoms is a manifestation of regeneration of the intervertebral disc - the DGU phenomenon. The control group with classic electrotherapy procedures experienced a transient improvement in health during the first 3-4 weeks. Then, the health condition changed harmoniously from the image of a painful condition before inclusion in the therapeutic block with periods of subjective good health. In the first (experimental) group, a growth of the intervertebral disc was observed in $76 \%$ of the patients, i.e., the DGU phenomenon was proven in 22 patients (volume of the intervertebral disc increased by more than $10 \%$ ), in $24 \%$ (4) of the patients, the DGU phenomenon was proven after the impedance therapy, with the intervertebral disc volume not decreasing and DDD not progressing $(0-5 \%$ increase in intervertebral disc volume). In patients with a proven DGU phenomenon, not only the spine but also the large joints improved significantly. Overall, the statistically significant increase in intervertebral disc volume was reported to be $31 \%, p<0.000$, reduction in perception of pain after IT $p<0.000$, and the final neurological examination, i.e., the examination of the periosteal and tendon reflexes, showed statistically significant changes in the manifestation of PTR $p$ $<0.00,0$ (Tab. 2).

In the second (control) group, patients received a standard electrotherapy. Each of the treated patients had a reduced intervertebral disc volume after long-term treatment according to a rehabilitation plan. DDD progressed according to Kirkaldy-Willis degeneration (1). Overall, a statistically significant reduction in the intervertebral disc volume of $15 \%, \mathrm{p}<0.000$, was observed, and the final neurological examination did not specifically show statistically significant changes in PTR, $p>0.005$. In the control group, only a reduction in pain perception, $\mathrm{p}<0.000$, after standard therapy was noted (Tab. 3).

In the obtained structured data, there was a correlation between a regeneration induction and blood lactate levels at rest and during aerobic activity (Tabs 4 and 5).

\section{Discussion}

The aim of the randomized clinical trial was to determine the impact of innovative IT on improving health in the rehabilitation of DDD patients. The impact of IT on the growth of the intervertebral disc was investigated. Our results showed a positive impact of IT on increasing the volume of the intervertebral disc in the observed file, where the "disc grow-up" phenomenon (DGU) was confirmed by MRI - proof of the growth of the intervertebral disc based on the effect of impedance therapy. In the control group, no DGU phenomenon was observed. Conservative treatment including a resting regimen and adequate medical treatment and rehabilitation is effective in $85-90 \%$ of patients at the level of subjective symptoms. Surgical treatment is indicated in $10 \%$ of patients in whom radicular irritation persists or neurological deficit progresses during conservative treatment. Rare syndromes and progressive motor-radicular deficiency require an urgent surgical treatment. Despite the available treatment, the remaining 5-10\% of patients remained chronically affected, especially patients with back pain. Surgical treatment in patients with chronic back pain is usually of little success. It is indicated for significant functional disability or pain not responding to multidisciplinary conservative treatment. The prognosis of patients is influenced by the severity of the clinical manifestation, the possibility of providing a rapid adequate treatment and psycho-socio-economic factors. Degenerative changes in the spine as a disease of modern civilization have so far been treated with medication or non-drug treatment, but have never been eliminated $(9,13)$.

\section{Based on our findings, we make the following conclusions}

It can be considered that the theory that degenerative spine changes are irreversible has been disproven by the impact of impedance therapy.

The basis of impedance therapy itself is the ability to influence the lost regenerative abilities of the organism. We called this phenomenon the DGU phenomenon and it has been proven in 1123 patients $(6,7)$.

Impedance therapy as a drug-free therapy for degenerative disc disease is becoming a medical procedure with an objectively measurable outcome of patient recovery.

\section{Conclusion}

In the randomized clinical trial, the effect of IT with SEI on the growth of the intervertebral disc in the experimental group of patients with DDD was observed. In the control group with standard electrotherapy, the manifestation of regeneration of the 
137-142

intervertebral disc, DGU, was not observed. The presented IT method opens a new perspective for the treatment and prognosis of degenerative changes in the spine. The results of impedance therapy in patients with degenerative disc diseases are the reason why we offer this innovative treatment process to other health care facilities with an objective measurable outcome.

\section{References}

1. Bertilson BC, Bring J, Sjoblom A, Sundell K, Strender LE. Interexaminer reliability in the assessement of low back pain using the Kirkaldy Willis classification. Eur Spine J 2006; 15: 1695-1703,

2. Choroš R. Integrovaný mikroelektródový systém, 2011, 50 p.

3. Deyo RA, Weinstein JN. Primary care: Low back pain. N Engl J Med 2001; 344: 363-370.

4. Freburger JK, Holmes GM, Agans RP et al. The Rising Prevalence of Chronic Low Back Pain. Arch Intern Med 2009; 169 (3): 251-258.

5. Jensen MP, Karoly P, O'Riordan EF, Bland F, Burns RS. The subjective experience of acute pain. An assessment of the utility of 10 indices. Clin J Pain 1989; 5 (2): 153-159. doi:10.1097/00002508-19890600000005. PMID 2520397

6. Kostka P. Impedančná terapia, 2017, 180 p.
7. Kostka P et al. Impedančná terapia II - aplikácia v medicínskej praxis. 2019, 204 p.

8. Martin BI, Deyo RA, Mirza SK et al. Expenditures and health status among adults with back and neck problems. J Amer Med Ass 2008; 299: 656-664.

9. Mcculloch JA, Young PH. Essentials of spinal microsurgery. Philadelphia: Lippincott-Raven, 1998.

10. Paulo Amorim HJ, De Moraes TF, Azevedo F, Da Silva JVL. In Vesalius: Software Livre de Imagens Medicas. www.researchgate.net/publication/260322057_InVesalius_Software_Livre_de_Imagens_Medics 2014

11. Rubin DI. Epidemiology and risk factors for spine pain. Neurol Clin 2007; 25 (2): 353-371.

12. Trávničková H. Kožní odpor a psychogalvanický reflex, 2012, 40 p.

13. Wheeler AH. Low Back Pain and Sciatica. eMedicine [online] (Feb 03. 2016). In: Berman SA. Medscape, (cit 20.12.2016), http://emedicine. medscape.com/article/1144130-overview\#a3.

14. WMA 2000. WMA Declaration of Helsinki - Ethical Principles for Medical Research Involving Human Subjects, WMA. In 52nd WMA General Assembly, Edinburgh, Scotland, October 2000. (cit. 2017-01-15). Dostupné na internete: www.samawomenshealth.in/wma-declaration-ofhelsinki- ethical-principles-for-medical-research-involving-human-subjects/. 